\title{
A new negative control gene for amino acid biosynthesis in Saccharomyces cerevisiae
}

\author{
Rachel C. Skvirsky*, Miriam L. Greenberg* ${ }^{*}$, Paul Louis Myers, and Helen Greer \\ Department of Cellular and Developmental Biology, Harvard University, Cambridge, MA 02138, USA
}

Summary. Enzyme levels in multiple amino acid biosynthetic pathways in yeast are coregulated. This control is effected largely at the transcriptional level by a number of regulatory genes. We report the isolation and characterization of a new negative regulatory gene, $G C D 4$, for this general control system. GCD4 mutations are recessive and define a single Mendelian gene on chromosome III. A gcd4 mutation results in resistance to different amino acid analogs and elevated, but fully inducible, mRNA levels of genes under general control. Epistasis analysis indicates that $G C D 4$ acts more directly than the positive regulators $G C N 1, G C N 2, G C N 3$ and $G C N 5$, but less directly than $G C N 4$, on the transcription of the amino acid biosynthetic genes. These data imply that GCD4 is a negative regulator of the positive effector, $G C N 4$. Although GCD4 occupies the same position relative to the $G C N$ genes as other $G C D$ genes, it produces a unique phenotype. These results illustrate the diversity of function of negative regulators in general control.

Key words: Gene expression - Transcriptional regulation - Yeast general control $-G C D$ genes

\section{Introduction}

The eukaryotic cell must respond to a variety of environmental changes with appropriate alterations of its metabolism. This response is often effected by the selective expression of a specific subset of the genome. In Sac-

Present addresses:

* Department of Biology, Simmons College, Boston, MA 02115, USA

** Department of Biological Chemistry, University of Michigan, Ann Arbor, MI 48109, USA

Offprint requests to: $\mathrm{H}$. Greer charomyces cerevisiae, starvation for a single amino acid results in increased transcription of amino acid biosynthetic genes in multiple amino acid biosynthetic pathways (Penn et al. 1983; Silverman et al. 1982; Struhl and Davis 1981; Zalkin and Yanofsky 1982). For example, limitation of histidine elicits derepression of genes in at least the histidine, arginine, tryptophan, leucine, isoleucine-valine and lysine biosynthetic pathways (Niederberger et al. 1981; Wolfner et al. 1975). This coregulation is called general control of amino acid biosynthesis (Delforge et al. 1975). Derepression occurs within $5 \mathrm{~min}$ of the imposition of amino acid starvation and is main. tained until the starvation condition is removed (Penn et al. 1984). The response is not coordinate; all of the enzymes of a given amino acid pathway may not be derepressed, and those that are may be derepressed to varying extents (Hsu et al. 1982; Miozzari et al. 1978). With the exceptions of the arginine and leucine biosynthetic pathways, no specific amino acid pathway control has been shown to exist in Saccharomyces.

The coregulated derepression of amino acid biosynthetic genes is mediated by an array of cis- and transacting regulatory elements. As expected for transcriptionally coregulated genes, they possess common $5^{\prime}$ regulatory structures. All genes under general control thus far examined are preceded by at least one copy of a 6 base pair $(\mathrm{bp})$ consensus sequence (Donahue et al. 1983; Hinnebusch and Fink 1983a; Struhl and Davis 1981; Zalkin and Yanofsky 1982). Deletion and gene fusion experiments have shown that the $6 \mathrm{bp}$ consensus sequence is required for derepression, probably acting as the site of interaction with a positive regulatory element (Donahue et al. 1983; Silverman et al. 1982). The $5^{\prime}$ regions of genes under general control also contain transcribed open reading frames which are involved in regulating the kinetics of derepression (Greer et al. 1982; B. Hauge and $\mathrm{H}$. Greer, in preparation).

Two classes of trans-acting regulatory genes mediate the general control response. Nine genes, GCN1-GCN9, 
that behave in a formal sense as positive regulatory elements, have been identified and characterized (Penn et al. 1983; Schurch et al. 1974; Wolfner et al. 1975; M. Greenberg, P. Myers, R. Skvirsky and H. Greer, submitted). ( $G C N$ is the new nomenclature and denotes general control nonderepressed. $G C N 1=N D R 1$ or $A A S 103, G C N 2$ $=N D R 2, A A S 1$ or $A A S 102, G C N 3=A A S 2, G C N 4=$ $A A S 101$ or $A A S 3$, and $G C N 5=A A S 104$.) $G C N 1, G C N 3$ and $G C N 4$ are necessary for the initiation of amino acid biosynthetic gene mRNA elevation (Penn et al. 1984). $G C N 2$, which is itself under general control, is required for the long term maintenance of mRNA elevation (Penn et al. 1984). GCN6 and $G C N 7$ positively regulate the expression of GCN4 at the transcriptional level (M. Greenberg, P. Myers, R. Skvirsky, and H. Greer, submitted). In addition, GCN4 is translationally regulated; under derepressing conditions, the $G C N 4$ transcript becomes translationally activated (Thireos et al. 1984). Thus, a number of positive regulatory genes act in diverse ways to derepress the amino acid biosynthetic genes.

When grown on medium containing all amino acids, the amino acid biosynthetic genes are expressed at a low basal level (Penn et al. 1983). Genes that function at least formally as negative regulators act to limit expression of the amino acid biosynthetic genes under these repressing conditions. These regulatory genes are called $G C D$ (for general control derepressed), and their mutational inactivation results in constitutive production or over-production of the genes under general control. Five such genes, $G C D 1, G C D 2, G C D 3, G C D 5$ and $G E N^{c}$ have been reported $(G C D 1=T R A 3$ and $G C D 2=C D R 1)$. A mutant allele of $G C D 1$ was isolated by its resistance to triazole alanine, an analog of histidine (Wolfner et al. 1975). The gcdl mutation results in an increase in amino acid biosynthetic enzyme activity and temperature sensitivity for growth on complete medium. However, $G C D 1$ has recently been shown to affect overall protein synthesis in the cell (D. Hill and K. Struhl, personal communication), and thus it may have only an indirect effect on the general control system. A mutation in $G C D 2$ was recovered as a tryptophan analog resistant revertant of gcnl (Miozzari et al. 1978). It shows increased tryptophan biosynthetic enzyme activity, but further characterization has not been reported. The $G C D 3$ gene may be a key negative regulator of the system. A $g c d 3$ mutation causes derepression by increasing transcription of the amino acid biosynthetic genes (P. Myers, R. Skvirsky, M. Greenberg and H. Greer, submitted). This increase occurs because the GCD3 gene is a negative regulator of transcription of the positive regulatory $G C N 4$ gene. $G C D 5$ acts post-transcriptionally to increase amino acid biosynthetic enzyme activity (M. Greenberg, P. Myers, R. Skvirsky and H. Greer, submitted). Mutations in another gene, $G E N^{c}$, cause increased levels of arginine biosynthetic enzyme activity only under certain repressing growth conditions (Messenguy 1979).

This paper discusses mutations in a new regulatory gene, $G C D 4$, which were recovered in the same extensive search for negative regulatory genes undertaken in our laboratory that had identified the $G C D 3$ and $G C D 5$ genes (P. Myers, R. Skvirsky, M. Greenberg and H. Greer, submitted). GCD4 is nonallelic to the other published $G C D$ mutations, and therefore identifies a new gene. A gcd4 mutation permits the cell to overcome amino acid starvation because it results in overproduction of the amino acid biosynthetic enzymes. As a result, gcd4 mutants are resistant to analogs of different amino acids. This overproduction has been shown to result from elevation of the RNA transcript levels of the amino acid biosynthetic genes, under both repressing and derepressing conditions. Analysis of the relationships between gcd4 and mutations in positive regulatory $G C N$ genes reveals that $g c d 4$ is epistatic to $g c n 1, g c n 2, g c n 3$, and gcn5, but that gcn4 is epistatic to gcd4. These results imply that, in a formal sense, the $G C N 1, G C N 2, G C N 3$ and $G C N 5$ gene products are negative regulators of the $G C D 4$ gene product, which in turn is a negative regulator of the positive effector, $G C N 4$.

\section{Materials and methods}

Media and genetic techniques. Yeast extract/peptone/dextrose (YEP), minimal, minimal + histidine (minimal/histidine), minimal + all 20 amino acids (minimal/AA), minimal $+10 \mathrm{mM}$ 3-amino-1,2,4-triazole (minimal/3AT), and minimal proline (uses proline instead of $\mathrm{NH}_{4}^{+}$as a nitrogen source) media, as well as genetic procedures, have been described (Greer 1981; Sherman et al. 1979). The presence of gcd4 was scored by growth of a his $4 \mathrm{C}-207^{\text {ts }} \mathrm{gcn} 2$ strain on minimal medium at $30^{\circ} \mathrm{C}$ or the growth of a $h i s 4 C-207^{\text {ts }}$ strain on minimal medium at $37^{\circ} \mathrm{C}$.

GCD4 mutant isolation. Independent YEP cultures of MP116-1B (MATa his4C-207 $7^{\text {ts }}$ gen2-15 ura3-52) or HG167-3B (MATa his $4 C-207^{\text {ts }}$ gcn2-101 trp 1-1) strains were grown overnight at $30^{\circ} \mathrm{C}$, washed with $\mathrm{H}_{2} \mathrm{O}$, plated on minimal + uracil medium $\left(2 \times 10^{8}\right.$ cells/plate), irradiated with U.V. light to $50 \%$ cell survival, and incubated for 4 days at $30^{\circ} \mathrm{C}$ to obtain $\mathrm{His}^{+}$ revertants. Gcd mutants were identified by a series of growth, genetic and molecular tests. The initial stages of the screening process are described in detail in (P. Myers, R. Skvirsky, M. Greenberg, and H. Greer, submitted). The two gcd 4 alleles obtained in this study are independent isolates.

RNA extraction and northern analysis. Cells from exponentially growing YEP cultures were inoculated into minimal/AA + uracil and minimal/3AT + uracil media and grown for approximately $12 \mathrm{~h}$ until mid-log phase. Care was taken that all cultures were of similar cell density at the time of harvest. RNA was extracted by the procedure in (Sripati and Warner 1978). Twenty micrograms of RNA was denatured and electrophoresed through a formaldehyde-agarose gel, blotted onto Gene Screen, and crosslinked with U.V. irradiation as described in (Church and Gilbert 1984; Maniatis et al. 1982). Filters were hybridized to $10^{7}$ counts of 
32P nick-translated DNA probe as described in (Maniatis et al. 1982), washed and exposed to Kodak XAR-5 X-ray film at $-70^{\circ} \mathrm{C}$ using a DuPont Cronex intensifying screen. Blots were dehybridized by boiling for $5 \mathrm{~min}$ in distilled $\mathrm{H}_{2} \mathrm{O}$; rehybridization of blots was performed as described in (Church and Gilbert 1984).

\section{Results}

\section{Mutant isolation}

Mutations in negative regulatory elements for general control of amino acid biosynthesis should result in con. stitutive production or over-production of the enzymes under general control. Mutations with this phenotype were selected by reverting the $\mathrm{His}^{-}$phenotype of a his $4 C-207^{\mathrm{ts}}$ gcn2 strain. His $4 C-207^{\mathrm{ts}}$ is a temperature sensitive (ts) lesion in the gene encoding histidinol dehydrogenase, the last step in histidine biosynthesis. The mutant enzyme possesses almost no cataly tic activity at the non-permissive temperature $\left(37^{\circ} \mathrm{C}\right)$ and reduced catalytic activity at the permissive temperature $\left(30^{\circ} \mathrm{C}\right)$. Strains harboring $h i s 4 C-207^{\text {ts }}$ are able to grow on minimal medium at $30^{\circ} \mathrm{C}$ by derepressing their amino acid biosynthetic genes, thus compensating, by increased transcription, for the impaired his $4 C-207^{\text {ts }}$ enzyme. If, in addition to his $4 C-207^{\text {ts }}$, a strain also carries a mutation in $G C N 2$ (one of the positive regulators), it will not be able to derepress and therefore will not grow on medium lacking histidine.

$\mathrm{His}^{+}$revertants were obtained by U.V. light mutagenesis of a his4C-207 ${ }^{\text {ts }}$ gcn2-15 ura3-52 strain (MP116-1B) and a his $4 C-207^{\text {ts }}$ gcn2-101 trpl-1 strain (HG167-3B). Gcd mutants represented a very small fraction of the revertants. Out of $7.6 \times 10^{10}$ cells used in the selections, $4.0 \times 10^{4}$ were $\mathrm{His}^{+}$revertants, and only 3 of these were gcd mutants (MP116-1B-1,-2 and HG167-3B-T34). Two of the $g c d$ mutations are in a previously undescribed gene which we have called $G C D 4$, and are discussed here. The third mutation, gcd5, is discussed elsewhere (M. Greenberg, P. Myers, R. Skvirsky and H. Greer, submitted). The mutant selections and genetic techniques that were used to identify gcd mutants are described in detail in (P. Myers, R. Skvirsky, M. Greenberg and H. Greer, submitted).

\section{Dominance-recessiveness}

Dominance-recessiveness was determined for the mutations conferring a $\mathrm{His}^{+}$phenotype in the two mutant strains MP116-1B-1 and MP116-1B-2 (gcd $^{-}$his4C-207 ${ }^{\text {ts }}$ gcn2-15 ura3-52). Each strain was mated with a his $4 C$ $207^{\text {ts }}$ gcn2-15 trp 1-1 strain, and the resulting diploids tested for growth on minimal medium at $30^{\circ} \mathrm{C}$. Both diploids failed to grow, indicating that the $\mathrm{gcd}^{-}$mutations in the original $\mathrm{His}^{+}$revertants were recessive to wild type, as expected of mutationally inactivated negative regulatory genes.

\section{Two alleles of GCD4}

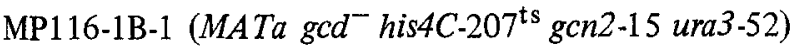

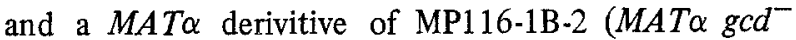
his $4 C-207^{\mathrm{ts}} \mathrm{gcn} 2-15 \operatorname{trp} 1-1$ ) were mated with each other. The resulting diploid grew on minimal medium at $30^{\circ} \mathrm{C}$. Since both alleles are recessive, this result indicates that the two gcd lesions are allelic. They have been designated gcd4-201 and gcd4-202, respectively. The allelism results were confirmed by mating a gcd4-201 his4C-207 $7^{\text {ts }}$ strain with a gcd4-202 his $4 C-207^{\text {ts }}$ strain in cross PLM392. Gcd4 partially suppresses the His phenotype of his $4 C$ $-207^{\text {ts }}$ on minimal medium at $37^{\circ} \mathrm{C}$, allowing intermediate growth (see below). Eleven tetrads from PLM392 yielded no spores that were $\mathrm{His}^{-}$at $37^{\circ} \mathrm{C}$ on minimal medium. If gcd4-201 and gcd4-202 segregated independently from each other, $11 \mathrm{His}^{-}$( his $4 C-207^{\text {ts }}$ ) spores would have been expected.

\section{2:2 Segregation}

Gcd4-201 his $4 C-207^{\text {ts }}$ gcn2-15 and gcd 4-202 his $4 C-207^{\text {ts }}$ gcn2-15 were each mated to a his $4 C-207^{\text {ts }}$ gcn2-15 strain in crosses PLM234 and PLM237, respectively, and tetrads analyzed to determine if gcd4 segregated as a single nuclear gene. Meiotic analysis of 20 tetrads from each cross indicated that the segregation pattern was $2 \mathrm{His}^{-}$spores $\left(G C D 4^{+}\right.$his $\left.4 C-207^{\mathrm{ts}} \mathrm{gcn} 2-15\right)$ to $2 \mathrm{His}^{+}$spores ( $\mathrm{gcd} 4$ his $4 C \cdot 207^{\text {ts }}$ gcn2-15). Thus, each strain contained a single mutation which was responsible for the $\mathrm{Gcd}^{-}$phenotype, and which segregated as a Mendelian gene.

\section{His $4 C^{t s}$ suppression}

In addition to overcoming the $\mathrm{His}^{-}$phenotype of a his $4 C-207^{\text {ts }} \mathrm{gcn} 2$ strain on minimal medium at $30^{\circ} \mathrm{C}$, the gcd 4 mutation was also shown to be able to suppress the His ${ }^{-}$phenotype of a his $4 C-207^{\text {ts }}$ strain on minimal medium at $37^{\circ} \mathrm{C}$. In crosses PLM418 and PLM488, gcd4-201 his $4 C-207^{\text {ts }}$ and gcd4-202 his $4 C-207^{\text {ts }}$ were each mated to a his $4 C-207^{\mathrm{ts}} \mathrm{gcn} 2-15$ strain. Each tetrad from these crosses contains 2 his $4 C^{\text {ts }}$ and 2 his $4 C^{\text {ts }}$ gcn2 spores. Some of the $h i s 4 C^{\mathrm{ts}} g c n 2$ spores grow on minimal medium at $30^{\circ} \mathrm{C}$ (contain gcd4). It was also observed that some of the his $4 C^{\mathrm{ts}}$ spores grow on minimal medium at $37^{\circ} \mathrm{C}$. Since his $4 C^{\text {ts }}$ strains cannot by themselves grow at $37^{\circ} \mathrm{C}$, this result implied that an additional mutation 
was responsible for this growth. If both the $30^{\circ} \mathrm{C}$ and $37^{\circ} \mathrm{C} \mathrm{His}{ }^{+}$phenotypes are due to the same mutation, 2 and only 2 spores of a tetrad would possess a His ${ }^{+}$phenotype (combining $30^{\circ} \mathrm{C}$ and $37{ }^{\circ} \mathrm{C}$ results). If the $37^{\circ} \mathrm{C}$ His ${ }^{+}$phenotype is due to one mutation, and the $30^{\circ} \mathrm{C}$ $\mathrm{His}^{+}$phenotype is due to a different mutation, tetrads with $0,1,2,3$ and $4 \mathrm{His}^{+}$spores would be obtained.

Suppression of the His phenotype was scored at $30{ }^{\circ} \mathrm{C}$ for his $4 \mathrm{C}-207^{\text {ts }}$ gcn2 -15 spores and at $37^{\circ} \mathrm{C}$ for his $4 C-207^{\text {ts }}$ spores. A total of 44 tetrads were scored and each possessed 2 and only 2 spores in which the His phenotype was suppressed, indicating that the $g c d 4 \mathrm{mu}$ tation is responsible for both types of suppression. Thus, the gcd4 mutation is able to derepress the temperaturesensitive $H I S 4 C$ enzyme to a level that is sufficient to permit this severely impaired enzyme to be functional at $37^{\circ} \mathrm{C}$.

\section{Linkage}

Since a reversion event at either $H I S 4 C$ or $G C N 2$ would have given a $\mathrm{His}^{+}$phenotype in the original mutant isolation, it was necessary to demonstrate that gcn 4 segregates independently from HIS4 and GCN2 (two genes which are themselves unlinked to each other). To this end, gcd4 his $4 C-207^{\text {ts }}$ gcn2-15 strains were crossed to wild type. If the $\mathrm{His}^{+}$phenotype conferred by $\mathrm{gcd} 4$ was due to a reversion event at HIS 4 or GCN2 (or both), his $4 C^{\text {ts }}$ gcn2 spores (His ${ }^{-}$on minimal medium at $30^{\circ} \mathrm{C}$ ) would not be recovered in these crosses. If $g c d 4$ is unlinked to HIS 4 and GCN2, half of the his $4 C^{\text {ts }} g c n 2$ spores should carry $g c d 4$ and exhibit a His ${ }^{+}$phenotype and half should carry $G C D 4$ and exhibit a His phenotype. Loose linkage of $g c d 4$ to either HIS4 or GCN2 would result in more $\mathrm{His}^{+}$spores $\left(\mathrm{gcd} 4 \mathrm{his} 4 \mathrm{C}^{\mathrm{ts}} \mathrm{gcn} 2\right)$ than His ${ }^{-}$spores $\left(\right.$his $4 C^{\text {ts }}$ gcn2).

Gcd4-201 his 4C-207 ${ }^{\text {ts }}$ gcn2-15 was crossed to wild type in crosses PLM372, PLM488, and PLM557. A total of 73 tetrads yielded 68 his $4 C^{\text {ts }} \mathrm{gcn} 2$ spores. Of these 68 spores, 49 were $\mathrm{His}^{+}\left(\mathrm{gcd} 4 \mathrm{his} 4 \mathrm{C}^{\mathrm{ts}} \mathrm{gcn} 2\right.$ ) and 19 were His ${ }^{-}\left(G C D 4^{+}\right.$his $\left.4 C^{\text {ts }} g c n 2\right)$ on minimal medium at $30^{\circ} \mathrm{C}$. Similar results were obtained with $\mathrm{gcd} 4-202$ in cross PLM409, where $g c d 4-202$ his $4 C-207^{\text {ts }}$ gcn2-15 was crossed to wild type. Twenty-nine tetrads yielded 19 his $4 C^{\text {ts }}$ gcn2 spores. Of these 19 spores, 15 were $\mathrm{His}^{+}$ and 4 were His ${ }^{-}$on minimal medium. The recovery of his $4 C^{\text {ts }}$ gcn2 spores that are His in both sets of crosses indicates that the His ${ }^{+}$phenotype of $g c d 4$ is not due to a reversion event at either HIS4 or GCN2. However, the preponderance of $\mathrm{His}^{+}$spores that contain his $4 C^{\text {ts }}$ and gcn2 indicates loose linkage of GCD4 to either HIS4 or GCN2. Further examination of the his $4 C^{t s} G C N 2^{+}$ spores from PLM488 on minimal medium at $37^{\circ} \mathrm{C}$ showed that GCD4 is loosely linked to HIS4. Of 34 such spores, 26 showed growth (His ${ }^{+}$) and 8 failed to grow (His ${ }^{-}$). An unlinked mutation would have resulted in an equal number of $\mathrm{His}^{+}$and $\mathrm{His}^{-}$spores. A reversion of the his $4 C-207^{\text {ts }}$ mutation to $H I S 4 C^{+}$would not yield any His spores at $37^{\circ} \mathrm{C}$. Thus, $g c d 4$ is loosely linked to HIS4C.

\section{Mapping}

The above crosses suggested that $G C D 4$ is loosely linked to HIS4. This conclusion was more rigorously tested in cross PLM370 in which a MATa gcd4-201 his $4 C-207^{\text {ts }}$ strain was mated to a $M A T \alpha$ leu2-1 strain. The map order of the relevant markers is HIS4-LEU2-centromere-MAT (located on chromosome III), with the distance between HIS4 and LEU2 being 17 map units and the distance between $L E U 2$ and $M A T$ being 35 map units (Mortimer and Schild 1980). Tetrad analysis of the PLM370 cross allowed the determination of the map distance between GCD4 and HIS4, as well as the order of GCD4 and HIS4 on chromosome III. The presence of the $g c d 4$ mutation was followed by its partial suppression of his $4 C^{\text {ts }}$ on minimal medium at $37^{\circ} \mathrm{C}$. Parental ditype (PD), nonparental ditype (NPD), and tetratype (T) tetrads were determined for each pair of markers and the distance between them calculated using the formula in (Perkins 1949):

map distance $=100 \times \frac{T+(6 N P D)}{2(P D+N P D+T)}$

In addition, the position of $G C D 4$ was confirmed by analyzing the meiotic data as a set of 3 -factor crosses in which the least frequent recombinant class corresponds to the double crossover class. GCD4 was thus determined to be 27 map units from HIS4, with the order being GCD4-HIS4-LEU2.

\section{Allelism to other GCD genes}

$G C D 4$ was shown to be non-allelic to $G C D 1, G C D 2$, and $G C D 3$. Since GCD4 is loosely linked to HIS4 on chromosome III, GCD3 is unlinked to HIS4 (P. Myers, R. Skvirsky, M. Greenberg and H. Greer, submitted), and $G C D 1$ is linked to $M E T 7$ on chromosome XV (G. Fink, personal communication), $G C D 4$ cannot be an allele of either of these $G C D$ genes. To determine if $G C D 4$ and $G C D 2$ are allelic, gcd4-201 his $4 C-207^{\text {ts }}$ gcn2-15 was mated to gcd2-101 in cross PLM758. Thirty seven tetrads yielded 37 his $4 C^{\text {ts }} g c n 2$ spores. Of these, 31 were $\mathrm{His}^{+}$and 6 were His ${ }^{-}$. The recovery of His spores indicates that $G C D 2$ and $G C D 4$ are not allelic. (GCD2 was shown to be epistatic to his $4 C-207^{\text {ts }} \operatorname{gcn} 2-15$ in a separate cross.) 


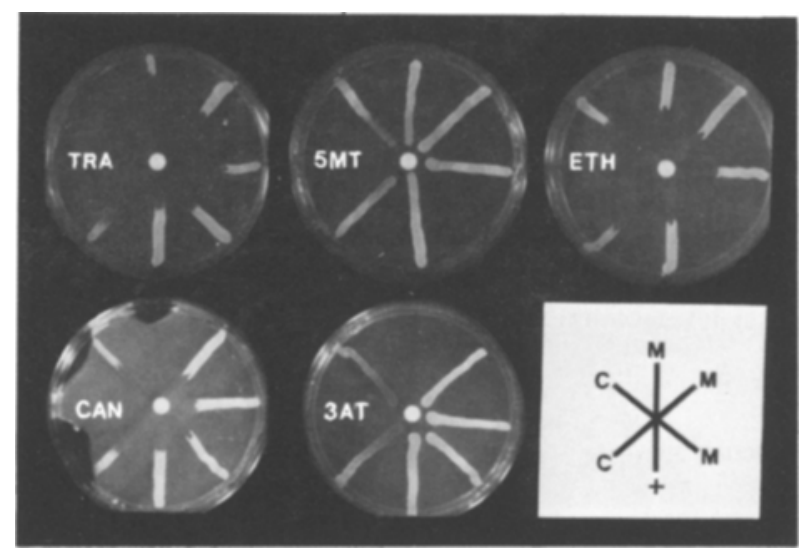

Fig. 1. Amino acid analog sensitivity of mutant and control strains. YEP cultures were washed with $\mathrm{H}_{2} \mathrm{O}$ and striped onto minimal proline + uracil + histidine medium. $10 \mu l$ of $50 \mathrm{mM}$ 5 -methyl-tryptophan (5MT), $20 \mu \mathrm{lof} 10 \mathrm{mg} / \mathrm{ml}$ ethionine (ETH), $20 \mu \mathrm{l}$ of $6 \mathrm{mg} / \mathrm{ml}$ canavanine (CAN), $40 \mu \mathrm{l}$ of $50 \mathrm{mM}$ triazole alanine (TRA) and $40 \mu \mathrm{l}$ of $1 \mathrm{M}$ 3-amino-1,2,4-triazole (3AT) solutions were each pipetted onto a sensi-disc in the center of a petri plate, and incubated for 2 days at $30^{\circ} \mathrm{C}$. The distance of the zone of growth inhibition from the disc indicates the degree of analog sensitivity. At the bottom of each plate is " + " which is wild type S288C (MAT $\alpha$ ). Proceeding in a clockwise direction: "C" is control PLM370-11A (his4C-207 ts ura3-52), "C" is control PLM370-27D (his4C-207 ${ }^{\text {ts }}$ ura3-52), "M" is mutant PLM370-16A (gcd4-201 his4C-207ts $u$ ra3-52), "M" is mutant PLM370-12B (gcd4-201, his4C-207's ura3-52), and " $M$ " is $\mathrm{mu}-$ tant PLM370-8C (gcd4-201 his4C-207 ${ }^{\text {ts }}$ ura3-52)

\section{Epistasis to GCN genes}

The positive regulatory $G C N$ genes have been shown to control diverse aspects of the derepression response. It is thus likely that the negative regulatory $G C D$ genes interact differently with the various $G C N$ genes. The $g c d 4$ mutations were isolated by their suppression of $g c n 2$ (i.e., gcd4 is epistatic to gcn2). In order to better understand the interactions of some of the general control regulatory genes, a gcd4-201 his $4 C-207^{\mathrm{ts}}$ strain was mated to a set of strains, each of which carried a mutation in one of four other $G C N$ genes. Meiotic analysis was performed and the $h i s 4 C^{\text {ts }} g c n$-x spores were identified by complementation to the appropriate tester strains. About $70 \%$ of these spores carry gcd4-201 and about $30 \%$ carry the wild type allele (GCD4 is loosely linked to HIS4). If $g c d 4-201$ is epistatic to a particular gcn mutation, then spores of the genotype gcd4-201 his $4 C^{\text {ts }}$ gcn-x will grow on minimal medium at $30^{\circ} \mathrm{C}$ (His ${ }^{+}$ phenotype); if $g c d 4$ is not epistatic, they will not grow (His ${ }^{-}$phenotype). Crosses of gcd4-201 his $4 C-207^{\text {ts }}$ to gcnl-15, gcn3-101, and gcn5-1 all resulted in his $4 C^{\text {ts }}$ $g c n$-x spores capable of growth on minimal medium at $30^{\circ} \mathrm{C}$. Thus, gcd4-201 can overcome the starvation imposed by a $g c n 1, g c n 3$ or gcn 5 mutation. However, it cannot overcome the starvation imposed by gcn $4-1$. Cross PLM676 ( gcd4-201 his4C-207 $7^{\mathrm{ts}} \times$ gcn4-1) yielded

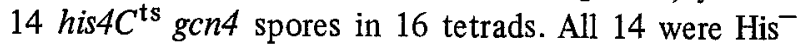
on minimal medium at $30^{\circ} \mathrm{C}$. Thus, gcd4-201 is epistatic to $g c n 1, g c n 2, g c n 3$, and $g c n 5$ but is not epistatic to gcn4.

\section{Amino acid analog resistance}

Amino acid analogs act in various ways to starve cells for a particular amino acid. Wild type cells can overcome the deleterious effects of certain concentrations of analogs by derepressing the corresponding amino acid biosynthetic pathway (as well as other amino acid biosynthetic pathways) via the general control system. Analog sensitivity was tested in $\operatorname{gcd} 4-201$ his $4 C-207^{\text {ts }}$ and his $4 C-207^{\text {ts }}$ strains from cross PLM370 (gcd4-201 his $4 C-207^{\text {ts }} \times$ leu2-1). As shown in Fig. 1, gcd4-201 confers resistance to 5-methyl-tryptophan (a tryptophan analog), canavanine (an arginine analog), ethionine (a methionine analog), and triazole alanine and 3-aminotriazole (both histidine analogs). These results suggest that the gcd4-201 mutation results in increased enzyme activity in at least the tryptophan, arginine, methionine, and histidine pathways, strongly implicating it in the general control system.

\section{mRNA levels}

Northern blot analysis indicates that the ged4-201 phenotype is due to an increase in the steady state mRNA levels of genes under general control. Blots of RNA extracted from wild type, the parental strain MP116-1B (his $4 C-207^{\text {ts }}$ gcn2-15), and the original mutant isolate MP116-1B-1 (gcd4-201 his4C-207 ${ }^{\text {ts }}$ gcn2-15), grown under repressing and derepressing conditions, were hybridized with a probe containing the HIS 4 and $U R A 3$ sequences. (The $U R A 3$ gene, which encodes orotidine-5'phosphate decarboxylase, an enzyme for uracil biosynthesis, is not under general control. It is used as an internal standard for the amount of RNA in each lane.) As seen in Fig. 2, the parent has lower levels of HIS4 RNA than wild type under both repressing and derepressing conditions. This result is due to the presence of gcn2 in the parent. In the $g c d 4$ mutant, the level of HIS4 RNA is clearly higher than in the parent under both repressing and derepressing conditions; in fact, the level of HIS4 RNA is restored to the HIS4 wild type level. Steady state RNA levels were also determined for another gene under general control, $A R G 4$ (encodes arginosuccinate lyase in the arginine biosynthetic pathway) (Messenguy 1979). ARG4 expression is low in the parent, again due to the presence of $g c n 2$. In the mutant, both repressed 


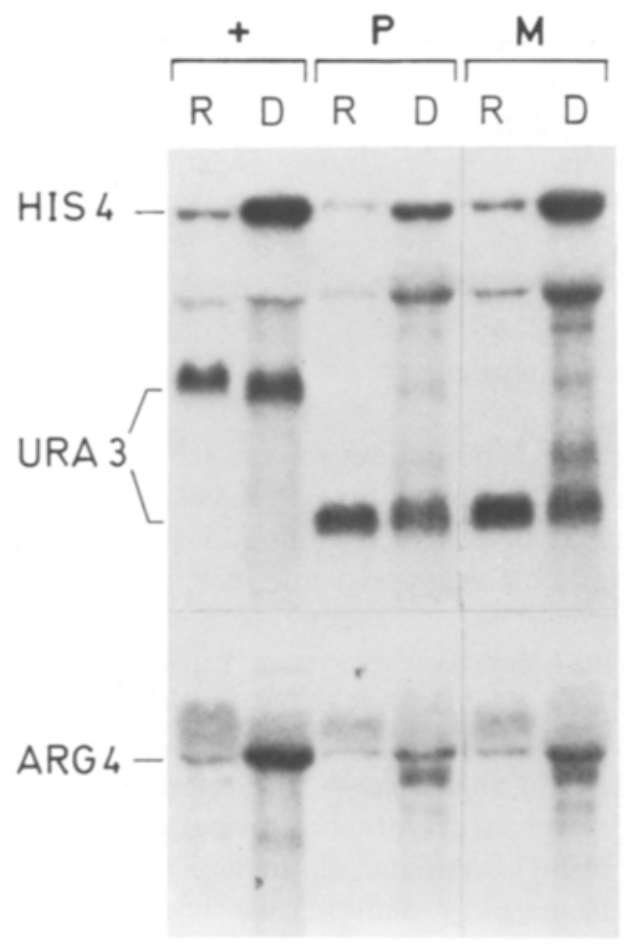

Fig. 2. Northern blot analysis of mutant and control strains. RNA was prepared from cell cultures grown at $30^{\circ} \mathrm{C}$ under repressing conditions ( $R$ ) in minimal/AA + uracil medium and derepressing conditions (D) in minimal/3AT + uracil medium, electrophoresed, blotted and hybridized with a ${ }^{32}$ P-labelled probe containing the yeast HIS 4 and URA3 genes (pBH21B; B. Hauge and H. Greer, unpublished). This same blot was dehybridized and rehybridized with a ${ }^{32}$ P-labelled probe containing the yeast ARG4 gene (pYE511; Clarke and Carbon 1978). "+" is wild type S288C (MAT $\alpha)$, "P" is the parent MP116-1B (MATa his 4C-207ts gcn2-15 ura3-52) and "M" is MP116-1B-1 (MATa gcd4-201 his 4C-207ts gcn2-15 ura3-52). The extent of hybridization with the URA3 gene was used to normalize the amount of RNA within each lane. The ura3-52 mutation, present in the P and $\mathrm{M}$ strains, is a chromosomal rearrangement (M. Rose, personal communication), and thus the URA3 RNA runs at a different position on the gel in these strains as compared to the wild type strain. The unidentified band in the top panel corresponds to HIS4 degradation products which comigrate with the $18 \mathrm{~S}$ ribosomal RNA band

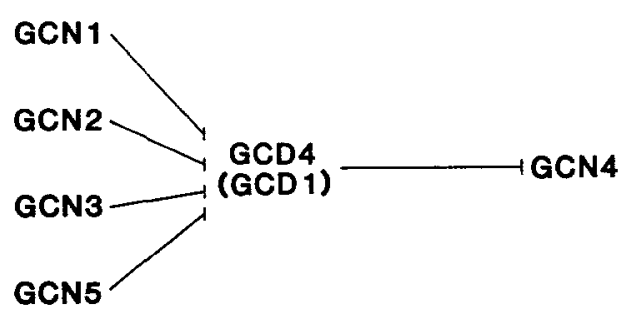

Fig. 3. Epistatic relationships of general control regulatory genes. - indicates negative regulation. Note: GCD1 occupies the same position relative to the $G C N 1-G C N 4$ genes as does GCD4 (it was not tested to GCN5) (Hinnebusch and Fink 1983b) and derepressed levels of $A R G 4$ RNA are increased over the parent.

These results indicate that $g c d 4-201$ results in an increase in the steady state mRNA levels of at least two genes under general control. The ratio of repressed to derepressed in $g c d 4-201$ is approximately the same as in wild type; thus, gcd4-201 appears to be elevating the basal level of amino acid biosynthetic gene expression.

\section{Discussion}

The expression of amino acid biosynthetic genes in yeast is mediated by a complex interaction of regulatory elements. Present understanding of the functioning of this system is limited. A more comprehensive view requires the identification and characterization of all major regulatory genes of the system. To this end, we conducted an extensive search for new $G C D$ genes that behave as negative regulators of amino acid biosynthetic gene expression. Previous searches for negative regulators relied on analog resistance as a selective technique (Miozzari et al. 1978; Wolfner et al. 1975). We have employed a different selection based on a $g c d$ mutation overcoming the $g c n$ dependent His ${ }^{-}$phenotype of a his $4 C^{\text {ts }}$ gcn strain. Three previously unidentified negative regulatory genes were recovered by this selection (P. Myers, R. Skvirsky, M. Greenberg and H. Greer, submitted). Two independent mutations in one of these genes, $G C D 4$, are described in this paper.

A gcd4 mutation results in inducible overproduction of amino acid biosynthetic gene activity. This overproduction results from an increase in the steady state RNA levels of amino acid biosynthetic genes. Epistasis studies indicate that a $g c d 4$ mutation is epistatic to mutations in the positive regulatory $G C N 1, G C N 2, G C N 3$, and GCN5 genes, i.e., a strain containing the double mutations $\mathrm{gcd} 4 \mathrm{gcn}^{-}$displays the derepressed phenotype of the single $g c d 4$ mutation. Thus, in the absence of wild type $G C D 4$ product, the $G C N 1, G C N 2, G C N 3$ and $G C N 5$ products are not needed for amino acid biosynthetic gene expression. The double mutant $\operatorname{gcd} 4 \mathrm{gcn} 4$, on the other hand, possesses the repressed phenotype of a gcn 4 mutant. These results suggest that GCD4 acts more directly than $G C N 1, G C N 2, G C N 3$, or $G C N 5$, but that GCN4 acts more directly than GCD4. This relationship is shown in Fig. 3. In this model of regulatory interaction, $G C N 1, G C N 2, G C N 3$, and $G C N 5$ are negative regulators of $G C D 4$, and $G C D 4$ is a negative regulator of $G C N 4$. The designation of negative or positive is derived from the known phenotypes of the mutants and their epistatic relationships. Thus, since $G C N 1$ is known to be ultimately a positive regulator of amino acid biosynthetic gene expression, and it is also known to act prior to $G C D 4$, it is depicted as a negative regulator of a negative 
regulator. While this scheme correctly reflects the data, it should be viewed with some qualification. The general control response is determined by many regulatory genes, only a few of which are sufficiently well characterized to be included in a model at this point.

Thus, to date, six negative regulatory genes of general control have been identified, $G C D 1, G C D 2, G C D 3$, $G C D 4, G C D 5$ and $G E N^{c}$. The phenotypes of these genes are diverse. A gcdl mutation results in constitutive derepression of genes under general control and also, perhaps primarily, greatly reduces overall cellular protein synthesis. Mutational inactivation of $G C D 2$ leads to derepression, but does not interfere with protein synthesis as does GCD1 inactivation. A gcd3 lesion results in a partially constitutive increase in amino acid biosynthetic gene mRNA. $G C D 3$ is also a negative regulator of $G C N 4$ transcription. Of the general control regulators thus far examined, $G C D 5$ is the only one to act post-transcriptionally. A $\mathrm{gen}^{c}$ mutation, unlike lesions in other general control negative regulators, results in amino acid biosynthetic enzyme activity only under certain repressing growth conditions. As this paper shows, $G C D 4$ represents yet another phenotypically unique negative regulator. Mutations of $G C D 4$ result in fully inducible over-production of mRNAs under general control, and preliminary data indicate that, unlike $g c d 3$ mutations, they do not affect GCN4 transcription. The diverse phenotypes of these genes demonstrate the complexity of negative regulation in general control.

Acknowledgements. We thank Mark Ganem for assistance in the isolation and analysis of these mutants. This work was supported by National Science Foundation Grant DCB-84-09701 to H. G. R. C. S. is a recipient of an American Cancer Society Postdoctoral Fellowship and M. L. G. is a recipient of a postdoctoral fellowship from the Medical Foundation, Inc.

\section{References}

Church G, Gilbert W (1984) Proc Natl Acad Sci USA 81:19911995

Clarke L, Carbon J (1978) J Mol Biol 120:517-532
Delforge J, Messenguy F, Wiame JM (1975) Eur J Biochem 57: 231-239

Donahue T, Daves R, Lucchini R, Fink G (1983) Cell 32:89-98

Greer H (1981) Mechanisms of yeast genetics. In: Holaender A (ed) Trends in the biology of fermentations for fuels and chemicals. Plenum Press, New York, pp 217-242

Greer H, Penn M, Hauge B, Galgoci B (1982) Control of amino acid biosynthesis in yeast. In: Esposito $M$ (ed) Recent advances in yeast molecular biology: recombinant DNA. University of California Press, Berkeley, pp 122-142

Hinnebusch A, Fink G (1983a) J Biol Chem 258:5238-5247

Hinnebusch A, Fink G (1983b) Proc Natl Acad Sci USA 80: $5374-5378$

Hsu Y-P, Kohlhaw G, Niederberger P (1982) J Bacteriol 150: 969-972

Maniatis T, Fritsch E, Sambrook J (1982) Molecular cloning: a laboratory manual. Cold Spring Harbor Laboratory, Cold Spring Harbor, NY

Messenguy F (1979) Mol Gen Genet 169:85-95

Miozzari G, Niederberger P, Hutter R (1978) J Bacteriol 134: $48-59$

Mortimer R, Schild D (1980) Microbiol Rev 44:519-521

Niederberger P, Miozzari G, Hutter R (1981) Mol Cell Biol 1: 584-593

Penn M, Galgoci B, Greer H (1983) Proc Natl Acad Sci USA 80: 2704-2708

Penn M, Thireos G, Greer H (1984) Mol Cell Biol 4:520-528

Perkins D (1949) Genetics 34:607-618

Schurch A, Miozzari J, Hutter R (1974) J Bacteriol 117:11311140

Sherman F, Fink G, Lawrence C (1979) Methods in yeast genetics: a laboratory manual. Cold Spring Harbor Laboratory, Cold Spring Harbor, NY

Silverman S, Rose M, Botstein D, Fink G (1982) Mol Cell Biol $2: 1212-1219$

Sripati E, Warner J (1978) Isolation, characterization and translation of mRNA in yeast. In: Prescott D (ed) Methods in cell biology, vol 20. Academic Press, New York, pp 61-81

Struhl K, Davis R (1981) J Mol Biol 152:535-552

Thireos G, Penn M, Greer H (1984) Proc Natl Acad Sci USA 81: 5096-5100

Wolfner M, Yep D, Messenguy F, Fink G (1975) J Mol Biol 96: 273-290

Zalkin H, Yanofsky C (1982) J Biol Chem 257:1491-1500

Communicated by G. Simchen

Received July 12 / September 9, 1985 